\title{
Percentages of CD4+CD161+ and CD4-CD8-CD161 + T Cells in the Synovial Fluid Are Correlated with Disease Activity in Rheumatoid Arthritis
}

\author{
Jinlin Miao, ${ }^{1}$ Kui Zhang, ${ }^{1}$ Feng Qiu, ${ }^{2}$ Tingting Li, ${ }^{3}$ Minghua Lv, ${ }^{1}$ Na Guo, ${ }^{1,4}$ \\ Qing Han, ${ }^{1}$ and Ping Zhu ${ }^{1}$ \\ ${ }^{1}$ Department of Clinical Immunology, Xijing Hospital, Fourth Military Medical University, Xian 710032, China \\ ${ }^{2}$ Department of Neurology, Chinese Navy General Hospital, Beijing 100048, China \\ ${ }^{3}$ Department of Geriatric Gastroenterology, Chinese People's Liberation Army General Hospital, Beijing 100853, China \\ ${ }^{4}$ Institute of Basic Medical Science, Xian Medical University, Xian 710032, China \\ Correspondence should be addressed to Ping Zhu; zhuping@fmmu.edu.cn
}

Received 17 October 2014; Accepted 1 December 2014

Academic Editor: Lifei Hou

Copyright (C) 2015 Jinlin Miao et al. This is an open access article distributed under the Creative Commons Attribution License, which permits unrestricted use, distribution, and reproduction in any medium, provided the original work is properly cited.

\begin{abstract}
Objective. CD161 has been identified as a marker of human IL-17-producing T cells that are implicated in the pathogenesis of rheumatoid arthritis (RA). This study aimed to investigate the potential link between the percentage of CD161+ T cells and disease activity in RA patients. Methods. Peripheral blood (PB) from 54 RA patients and 21 healthy controls was evaluated. Paired synovial fluid (SF) $(n=17)$ was analyzed. CD161 expression levels on CD4+, CD8+, and CD4-CD8- T cells were assessed by flow cytometry. Results. The percentage of CD4+CD161+ T cells in RA SF was higher than RA PB, and it was positively correlated with DAS28, erythrocyte sedimentation rate (ESR), and C-reactive protein (CRP). CD4-CD8-CD161+ T cell percentage was decreased in RA PB and was further reduced in RA SF, and its level in SF was inversely correlated with DAS28, ESR, and CRP. However, CD8+CD161+ $\mathrm{T}$ cell percentage was neither changed in RA PB and SF nor correlated with disease activity indices. Conclusion. An increased $\mathrm{CD} 4+\mathrm{CD} 161+\mathrm{T}$ cell percentage and a decreased CD4-CD8-CD161+ T cell percentage are present in RA SF and are associated with disease activity, and the accumulation of CD4+CD161+ T cells in SF may contribute to the local inflammation of RA.
\end{abstract}

\section{Introduction}

Rheumatoid arthritis (RA) is a systemic inflammatory disease characterized by joint inflammation of synovial tissue eventually leading to joint damage and functional disability. Multiple innate and adaptive effector cells, including macrophages, neutrophils, fibroblasts, $\mathrm{B}$ cells, and $\mathrm{T}$ cells, play important roles in the pathogenesis of RA [1]. T helper-type 17 (Th17) cells, a distinct subset of Th cells producing interleukin(IL-) 17 in humans, may be involved in the pathogenesis of autoimmune and chronic inflammatory disorders, including RA [2,3]. Numerous clinical studies including our data have demonstrated that the percentage of Th17 cells in RA patients was elevated and positively correlated with the degree of local and systemic disease activity [4-6]. Moreover, IL-17, the characteristic cytokine of Th17 cells, was implicated in the pathogenesis of RA [7]. IL-17A is a proinflammatory cytokine expressed in synovial membrane cultures of RA patients [8] and synovial tissue IL-17 is associated with more rapid joint damage progression in synergy with tumor necrosis factor(TNF-) $\alpha$ [9]. An enhanced expression of IL-17 has also been observed in the synovial fluid of RA patients $[8,10]$, and IL17 has become a new therapeutic target for mouse RA models and human RA [11].

CD161 is the human equivalent of mouse NK cell receptor P1A and constitutes a type II transmembrane glycoprotein with characteristics of the C-type lectin superfamily [12]. CD161 was one of the most upregulated genes in human Th17 cells compared to Th1 or Th2 cells and its expression is induced by RAR-related orphan receptor C (RORC), the Th17 lineage transcription factor $[13,14]$. In addition, human Th17 cells exclusively originate from CD4+CD161+ 
naive $\mathrm{T}$ cell progenitors, and CD161 is a novel surface marker for Th17 cells $[13,14]$. Moreover, Maggi et al. provided evidence that CD161 is a marker of all human IL17-producing $\mathrm{T}$ cell subsets, including CD3+CD4+CD8-, CD3+CD4-CD8+, and CD3+CD4-CD8- cells [14]. It has been reported that circulating $\mathrm{CD} 4+\mathrm{CD} 161+\mathrm{T}$ cells are increased in seropositive arthralgia patients but decreased in newly diagnosed RA patients [15]. Furthermore, this study showed that CD4+CD161+ T cells were enriched in synovial fluid $(\mathrm{SF})$, while $\mathrm{CD} 8+\mathrm{CD} 161+\mathrm{T}$ cells were not accumulated in SF of RA patients [15]. In fact, we have previously demonstrated that RA patients seemed to have higher percentages of circulating CD161+ cells in CD4+ T cells than healthy controls, but the difference did not reach statistical significance [16]. However, little was known about the percentages of CD161 expressing T cell subsets (including $\mathrm{CD} 3+\mathrm{CD} 4+, \mathrm{CD} 3+\mathrm{CD} 8+$, and CD3+CD4-CD8- cells) in blood and the local site of inflammation of RA patients and their potential link to disease activity.

Therefore, we explored the percentages of CD161 expressing $\mathrm{T}$ cell subsets in PB and SF of RA patients and assessed their correlations with the degree of disease activity.

\section{Materials and Methods}

2.1. Patients. Samples of peripheral blood (PB) were obtained from 54 RA patients and from 21 age- and sex-matched healthy controls. And synovial fluid (SF) samples were obtained from the knee joints of 17 patients with active RA. All patients fulfilled the 1987 revised criteria of the American College of Rheumatology [17]. Disease activity was assessed by the 28-joint disease activity score (DAS28) on the day of sample collection. Erythrocyte sedimentation rate (ESR) and C-reactive protein (CRP) were determined on the day of sample collection in the clinical laboratory. The study conforms to the recommendations of the Declaration of Helsinki. The Ethics Committee of Xijing Hospital approved this study, and the informed consent from all subjects was obtained.

2.2. Preparation of Mononuclear Cells. SF samples were treated with $20 \mu \mathrm{g} / \mathrm{mL}$ hyaluronidase (Sigma-Aldrich, St. Louis, MO, USA) for $30 \mathrm{~min}$ at $37^{\circ} \mathrm{C}$, and cells were then washed twice with phosphate-buffered saline. SF mononuclear cells (SFMCs) and PB mononuclear cells (PBMCs) were isolated from sodium heparinized whole blood and SF cell suspension samples using Ficoll-Paque density gradient centrifugation (GE Healthcare, Pittsburgh, PA, USA) by standard procedures.

2.3. Flow Cytometric Analysis of T Cell Surface Markers. The phenotypes of lymphocytes in PB and SF were determined using flow cytometry. Briefly, PBMCs and SFMCs were stained with the following fluorochrome conjugated monoclonal antibodies: fluorescein isothiocyanate- (FITC-) conjugated CD3 (SK7), peridinin chlorophyll protein- (PerCP-) conjugated CD4 (SK3), allophycocyanin- (APC-) conjugated CD8 (SK1), phycoerythrin- (PE-) conjugated CD161 (DX12), and isotype-matched control IgG antibodies (all from BD Biosciences, San Diego, CA, USA) for $30 \mathrm{~min}$ at room temperature, according to the manufacturer's instructions. Stained cells were analyzed using FACSCalibur flow cytometer (BD Biosciences), and data analysis was performed with Cell Quest software (BD Biosciences).

2.4. Statistical Analysis. Differences between groups were determined using the nonparametric Mann-Whitney test. Paired samples were compared using a Wilcoxon matched pairs signed rank sum test. Correlations were evaluated by nonparametric Spearman's correlation analysis. Data analyses were performed using GraphPad Prism version 5.0 (GraphPad Software, San Diego, CA, USA). For all tests, a two-sided $P$ value less than 0.05 was considered significant.

\section{Results}

3.1. Subject Basic Characteristics. Clinical characteristics of RA patients and healthy controls are illustrated in Table 1. Fifty-four patients with RA and 21 healthy controls (HC) were recruited, and synovial fluid (SF) samples were obtained from 17 active RA patients. There was no significant difference in age and gender between the three groups. In addition, disease duration, positive rate of RF and anti-CCP antibodies, and proportion of patients previously using medications were comparable between the group of total RA patients and the group of those patients with collected SF. In addition, ESR levels tended to be increased in RA patients with collected SF $(P=0.052)$, and CRP and DAS28 levels were significantly higher in RA patients with collected SF than in total RA patients $(P=0.032$ and $P=0.017$, resp. $)$.

3.2. Percentage of Circulating CD161+ T Cells in RA Patients and Healthy Controls. First, we assessed circulating CD3+ $\mathrm{T}$ cell subsets expressing the IL-17 producing cells marker CD161 in RA patients and HC, and representative examples of flow cytometric dot-plots are shown in Figure 1(a). The percentage of circulating CD4+CD161+ (22.19, 18.41-29.44\%) (median, interquartile range) and CD8+CD161+ cells (19.90, 16.26-29.74\%) in RA patients was not different from HC (20.34, 18.35-22.58\%, and 19.27, 17.19-24.27\%; $P=0.122$ and $P=0.675$, resp.) (Figures $1(\mathrm{~b})$ and $1(\mathrm{c})$ ), while the percentage of CD4-CD8-CD161+ cells was significantly lower in RA patients $(65.22,53.92-72.81 \%)$ than in $\mathrm{HC}(77.54,73.92-$ 82.14\%; $P<0.001$ ) (Figure 1(d)).

3.3. Percentage of CD161+ T Cells at the Site of Inflammation in RA. CD161 may function as an adhesion molecule and is involved in transendothelial migration [18, 19]. Then, the relative percentages of CD161 expression T cells in SF from patients with RA were assessed. The percentage of CD4+CD161+ cells in the RA SF was significantly increased (36.71,34.99-43.18\%) as compared to HC PB $(P<0.001)$, total RA PB $(P<0.001)$, and paired RA PB $(25.43,20.33-30.04 \%$; $P<0.001$ ) (Figures 1(b) and 2(a)), while a significantly lower percentage of CD4-CD8-CD161+ cells was observed in the RA SF $(35.50,31.49-40.45 \%)$ than in $\mathrm{HC} \mathrm{PB}(P<0.001)$, 

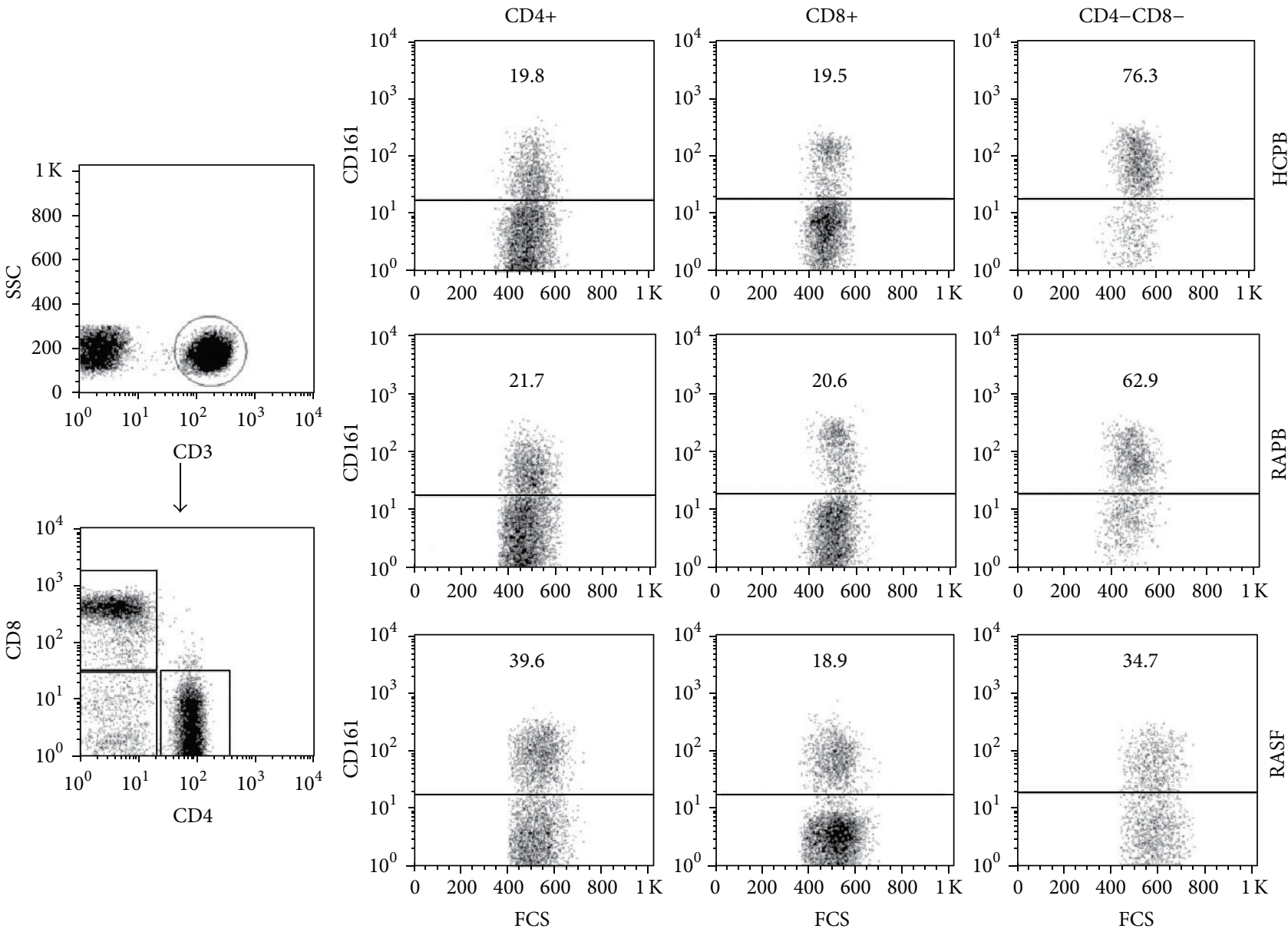

(a)

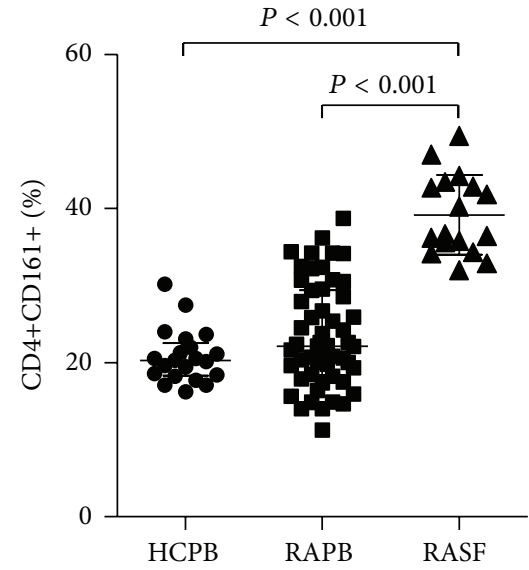

(b)

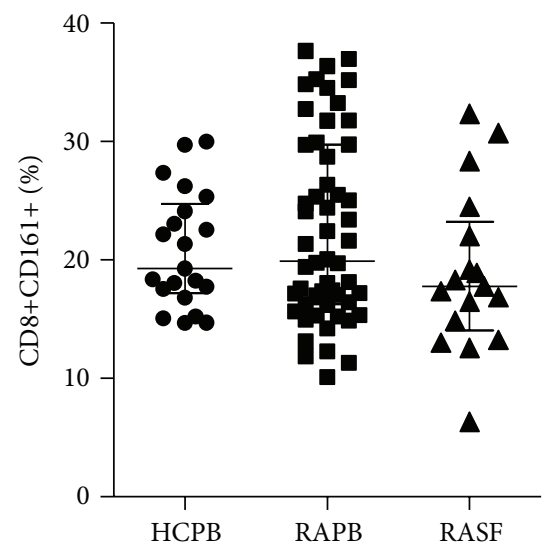

(c)

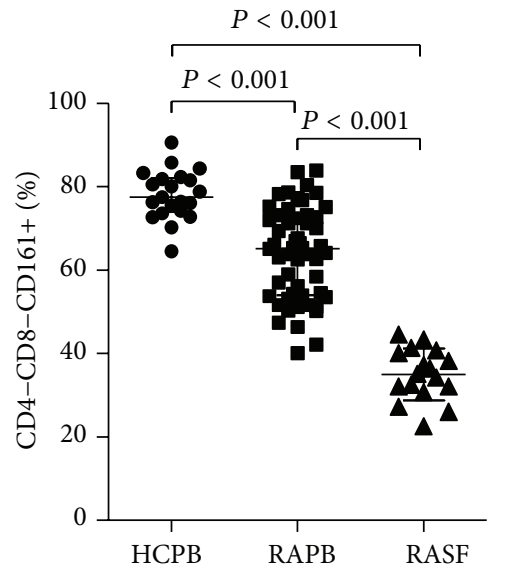

(d)

FIgure 1: Presence of CD161+ T cell subsets in RA patients and HC. (a) Flow cytometric dot-plots show gating strategy: CD3+ T cells were gated using side scatter profile and the expression of CD3; then CD4+, CD8+, and CD4-CD8- T cells were gated based on their expression of CD4 and CD8, and the CD161 expression levels in these T cell subsets were analyzed from representative HC peripheral blood (PB), RA PB, and RA synovial fluid (SF). Percentages of CD4+CD161+ (b), CD8+CD161+ (c), and CD4-CD8-CD161+ T cells (d) in HC PB, RA PB, and RA SF. Horizontal line indicates median value. $P$ values were assessed by Mann-Whitney $U$ test. 
TABLE 1: Characteristics of rheumatoid arthritis (RA) patients and healthy controls (HC).

\begin{tabular}{lccc}
\hline Characteristics & HC & RA & SF RA \\
\hline Number of patients & 21 & 54 & 17 \\
Age in years, median (IQR) & $45.0(34.0-53.0)$ & $46.5(36.5-54.8)$ & $40(74.1)$ \\
Female sex, $n$ (\%) & $15(71.4)$ & $66.0(11.8-115.5)$ & $13(76.5)$ \\
Disease duration, mo, median (IQR) & na & $38(70.4 \%)$ & $60.0(19.0-102.0)$ \\
Rheumatoid factor positive, $n(\%)$ & na & $40(74.1 \%)$ & $12(70.6 \%)$ \\
Anti-CCP positive, $n(\%)$ & na & $26.5(14.8-54.0)$ & $14(82.4 \%)$ \\
ESR, mm/hour, median (IQR) & na & $0.6(0.3-3.6)$ & $54.0(25.5-72.0)$ \\
CRP, mg/dL, median (IQR) & na & $4.5(2.6-5.7)$ & $3.1(0.5-5.7)^{*}$ \\
DAS28, median (IQR) & na & $7(13.0)$ & $5.4(4.5-6.1)^{*}$ \\
Systemic steroids, $n(\%)$ & na & $8(14.8)$ & $2(11.8)$ \\
NSAIDs, $n$ (\%) & na & $43(79.6)$ & $3(17.6)$ \\
DMARDs (excluding anti-TNF), $n(\%)$ & na & $8(14.8)$ & $13(76.5)$ \\
Anti-TNF- $\alpha$ therapy, $n(\%)$ & na & $3(17.6)$ \\
\hline
\end{tabular}

Values are presented as median (interquartile range) or number (percentage). SF, synovial fluid; IQR, interquartile range; Anti-CCP, anticyclic citrullinated peptide antibodies; ESR, erythrocyte sedimentation rate; CRP, C-reactive protein; DAS28, 28-joint disease activity score; NSAIDs, nonsteroidal antiinflammatory drugs; DMARDs, disease-modifying antirheumatic drugs; TNF- $\alpha$, tumor necrosis factor- $\alpha$; na, not applicable. ${ }^{*} P<0.05$ compared to RA patients.

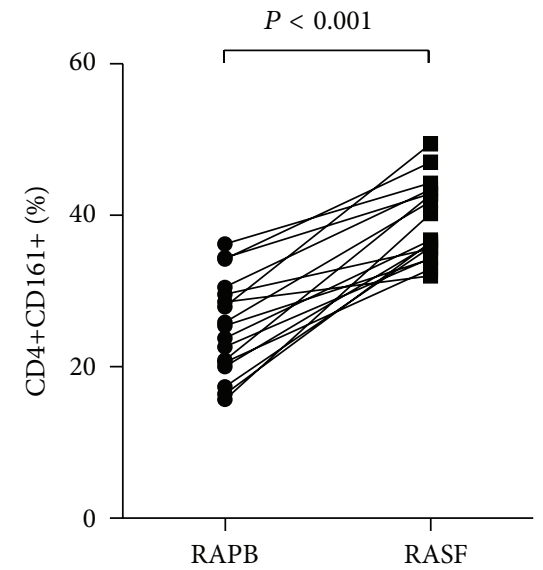

(a)

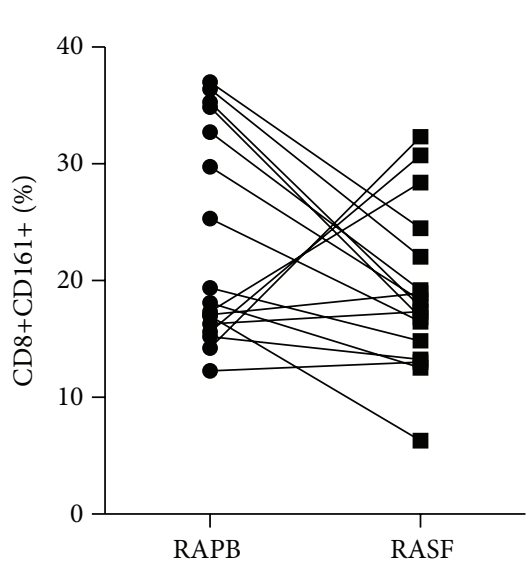

(b)

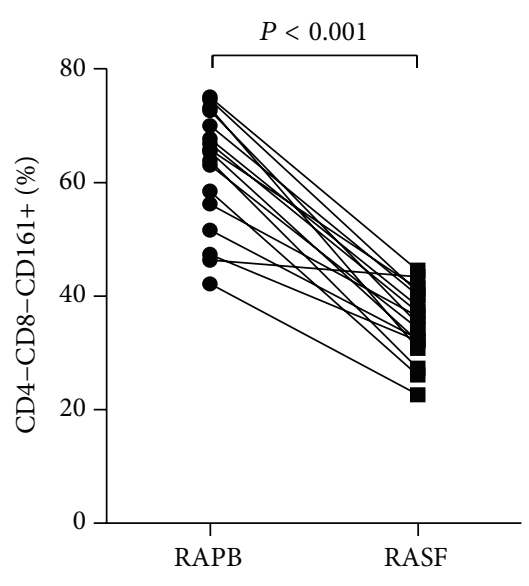

(c)

Figure 2: The percentages of CD4+CD161+ (a), CD8+CD161+ (b), and CD4-CD8-CD161+ T cells (c) in paired RA PB and SF samples are shown. $P$ values were assessed by Wilcoxon matched pairs signed rank sum test.

total RA PB $(P<0.001)$, and paired PB $(65.41,53.91-71.36 \%$; $P<0.001$ ) (Figures $1(\mathrm{~d})$ and $2(\mathrm{c})$ ). However, there were no significant differences in the percentage of CD8+CD161+ $\mathrm{T}$ cells among HC PB, total RA PB, RA SF (17.75, 14.06-23.27\%) and paired $\mathrm{PB}(18.12,15.96-33.80 \%)$ (all $P>0.05)$ (Figures 1(c) and 2(b)).

3.4. Correlations of CD161+ T Cells with Disease Activity in $R A$ Patients. Then, we assessed if the presence of CD161+ T cells in SF was correlated with systemic markers of disease activity (Table 2). The percentage of CD4+CD161+ cells in SF was positively correlated with DAS28 $(r=0.689, P=0.002)$, ESR $(r=0.569, P=0.017)$, and CRP levels $(r=0.679, P=0.003)$. In contrast, the percentage of CD4-CD8-CD161+ cells in SF was correlated inversely with DAS28 $(r=-0.671, P=0.003)$, ESR $(r=-0.632, P=0.007)$, and CRP levels $(r=-0.663$,
$P=0.004)$. However, no correlations were present between percentages of CD8+CD161+ cells in SF and DAS28 $(r=0.137$, $P=0.599)$, ESR $(r=-0.199, P=0.445)$, and CRP levels $(r$ $=0.074, P=0.779)$. Additionally, there were no correlations between systemic markers of disease activity and CD161+ T cells in PB (all $P>0.05$ ) (Table 2).

\section{Discussion}

Ample previous studies have indicated that Th17 cells and IL-17 critically contribute to the pathogenesis of RA [211]. Furthermore, not only CD4+ cells but also CD8+ and CD4-CD8- T cells that produce IL-17 express the CD161 on their surface, CD161 thus is considered a marker of all IL-17producing $\mathrm{T}$ cells [14]. Moreover, it has been reported that the expression of CD161 is maintained in the life cycle of 
TABLE 2: Correlations between percentages of CD161+ T cell subsets in RA synovial fluid (SF) and peripheral blood (PB) and DAS28, ESR, and CRP.

\begin{tabular}{lccccccc}
\hline & \multicolumn{2}{c}{ DAS28 } & & & ESR & & CRP \\
& $r$ & $P$ & $r$ & $P$ & $r$ & $P$ \\
\hline SF & & & & & & & \\
CD4+CD161+ & 0.689 & $\mathbf{0 . 0 0 2}$ & 0.569 & $\mathbf{0 . 0 1 7}$ & 0.679 & $\mathbf{0 . 0 0 3}$ \\
CD8+CD161+ & 0.137 & 0.599 & -0.199 & 0.445 & -0.074 & 0.779 \\
CD4-CD8-CD161+ & -0.671 & $\mathbf{0 . 0 0 3}$ & -0.632 & $\mathbf{0 . 0 0 7}$ & -0.663 & $\mathbf{0 . 0 0 4}$ \\
PB & & & & & & \\
CD4+CD161+ & 0.224 & 0.103 & 0.099 & 0.477 & 0.137 & 0.324 \\
CD8+CD161+ & 0.106 & 0.445 & 0.044 & 0.752 & 0.167 & 0.227 \\
CD4-CD8-CD161+ & -0.191 & 0.166 & -0.104 & 0.453 & -0.125 & 0.367 \\
\hline
\end{tabular}

human IL-17-producing T cells [13, 14, 18]. Therefore, CD161 expression may represent a way to detect IL-17-producing $\mathrm{T}$ cell ancestry in circulating and tissue infiltrating $\mathrm{T}$ cells.

We hypothesized that CD161 expressing T cells representing IL-17-producing $T$ cells act on the pathogenesis of $\mathrm{RA}$, and the levels of CD161 expression T cells in circulation or at inflammatory sites may be regulated prior to or after the development of inflammatory arthritis. Therefore, we assessed the percentage of circulating CD161 expression T cells in HC and RA patients first. Our results showed that the percentage of circulating CD4+CD161+ cells was not different from HC, as we previously reported [16]. However, conflicting data have been reported in a recent study which showed that patients with newly diagnosed RA had decreased levels of CD4+CD161+ cells [20]. Some of this variation may be due to the study cohort. The previous study recruited patients with newly diagnosed RA (with a mean duration of preceding symptoms of 10.2 months), while our cohort had a median disease duration of 66 months and thus may be more consistent with patients with established disease. In addition, this variation may be explained by treatment effects, as most of patients in our study were treated with DMARDs (Table 1), while the patients in the previous study were treatment-naïve [20].

CD161 may function as an adhesion molecule and thereby facilitates extravasation and tissue localization $[18,19]$. And a recent study reported that CD161 is a receptor expressed on different $\mathrm{T}$ cell subsets and may be involved in the pathogenesis of a given disease [21]. Hence, we investigated the performance of CD161 expressing $\mathrm{T}$ cells from the synovial fluid of RA patients as representative cells from the RA inflammatory site. Indeed, synovial fluid from active RA patients was found to be enriched in CD4+CD161+ T cells, which is in agreement with the data of a recent study [20]. Moreover, the percentage of CD4+CD161+ cells was positively correlated with DAS28, ESR, and CRP levels in SF of RA patients. These findings suggest that extravasation and migration of $\mathrm{CD} 4+\mathrm{CD} 161+\mathrm{T}$ cells to the joints may be facilitated by CD161 mediated adhesion [19] and indicate that CD4+CD161+ T cells, as Th17 precursor cells, may play a pathogenic role at the local site of inflammation in RA. Moreover, the proportions of CD4+CD161+ $\mathrm{T}$ cells in SF might reflect the degree of disease activity in RA patients.
Thus, we believe that further studies are needed to assess whether the CD4+CD161+ population was associated with joint damage progression, such as cartilage damage and bone erosion.

Meanwhile, levels of CD8+CD161+ cells and CD4-CD8-CD161+ cells in PB and SF were also examined in this study. Similar to a previous study [22], our data showed that the percentage of circulating CD8+CD161+ T cells in RA patients was not different from HC. In addition, no differences were observed between RA SF and paired RA PB or total RA PB of CD8+CD161+ T cells. Furthermore, there was no correlation between $\mathrm{CD} 8+\mathrm{CD} 161+\mathrm{T}$ cell percentage and disease activity in SF or PB of RA. This could be due to the relatively limited number of patients, but an intrinsic phenomenon is possible as well.

As reported in the literature, upon activation of the $\mathrm{T}$ cell receptor, a small number of $\mathrm{CD} 3+\mathrm{T}$ cells that are $\mathrm{CD} 4$ and CD8 double negative have the capacity to produce IL-17 [23]. Furthermore, IL-17-producing cells were only found in the CD161+ cell fraction of CD4-CD8 - cells, and CD4-CD8- T cells have the highest mRNA expression of RORC and IL-23R when compared to CD4+ and CD8+ T cells [14]. In addition, IL-17-producing CD4-CD8- T cells were expanded and involved in the pathogenesis of kidney damage and salivary gland damage in patients with systemic lupus erythematosus and Sjögren's syndrome $[24,25]$. However, we demonstrated that the percentage of circulating CD4-CD8-CD161+ cells, representing IL-17-producing CD4-CD8- T cell ancestry cells, was significantly decreased in RA patients when compared to HC. To our surprise, there was no correlation between circulating CD4-CD8-CD161+ cell percentage and the disease activity indices of RA. Furthermore, CD4-CD8-CD161+ cell percentage in RA SF was further decreased as compared to paired RA PB and total RA PB, and this reduction in SF was negatively correlated with DAS28, ESR, and CRP levels in RA patients. These findings suggest that the pathogenesis of RA may differ from that of other rheumatic diseases, and further investigations are needed to determine the mechanism for decreased CD4-CD8-CD161+ cell percentage and the performance of IL-17-producing CD4-CD8- T cells in RA patients. In addition, these results indicate that CD4+CD161+ and CD4-CD8-CD161+ T cells in the joint fluid may be considered as local parameters 
of joint inflammation, whereas CRP and ESR levels were regarded as systemic parameters of inflammation. More importantly, these data point towards a potentially important role for CD4+CD161+ and CD4-CD8-CD161+ T cells as regulators of joint inflammation and RA pathogenesis.

\section{Conclusion}

In conclusion, our results demonstrated that an increased percentage of CD4+CD161+ T cells and a decreased percentage of CD4-CD8-CD161+ T cells are present in SF of RA patients and correlate well with disease activity indices, and thus, may be involved in the local inflammation and clinical outcome of RA. These data suggest that CD4+CD161+ and CD4-CD8-CD161+ T cell levels in SF may reflect the degree of disease activity and joint inflammation in RA patients. Further studies are required to clarify the pathogenetic role of CD4+CD161+ and CD4-CD8-CD161+ T cells and investigate the mechanism for their change in RA.

\section{Disclosure}

Jinlin Miao, Kui Zhang, Feng Qiu, and Tingting Li are joint first authors.

\section{Conflict of Interests}

The authors declare that there is no conflict of interests regarding the publication of this paper.

\section{Acknowledgments}

This work was supported by grants from the Key Program of the National Natural Science Foundation of China (no. 81030058) and the National Natural Science Foundation of China (no. 81401338). This research was approved by Ethical Standards Committee of Xijing Hospital.

\section{References}

[1] I. B. McInnes and G. Schett, "The pathogenesis of rheumatoid arthritis," The New England Journal of Medicine, vol. 365, no. 23, pp. 2205-2219, 2011.

[2] H. Park, Z. Li, X. O. Yang et al., "A distinct lineage of CD4 T cells regulates tissue inflammation by producing interleukin 17," Nature Immunology, vol. 6, no. 11, pp. 1133-1141, 2005.

[3] F. Annunziato, L. Cosmi, F. Liotta, E. Maggi, and S. Romagnani, "Type $17 \mathrm{~T}$ helper cells-origins, features and possible roles in rheumatic disease," Nature Reviews Rheumatology, vol. 5, no. 6, pp. 325-331, 2009.

[4] H. Shen, J. C. Goodall, and J. S. Hill Gaston, "Frequency and phenotype of peripheral blood Th17 cells in ankylosing spondylitis and rheumatoid arthritis," Arthritis and Rheumatism, vol. 60, no. 6, pp. 1647-1656, 2009.

[5] D. Y. Chen, Y. M. Chen, H. H. Chen et al., "Increasing levels of circulating Th17 cells and interleukin-17 in rheumatoid arthritis patients with an inadequate response to anti-TNF- $\alpha$ therapy," Arthritis Research \& Therapy, vol. 13, no. 4, article R126, 2011.
[6] J. Miao, K. Zhang, M. Lv et al., "Circulating Th17 and Th1 cells expressing CD161 are associated with disease activity in rheumatoid arthritis," Scandinavian Journal of Rheumatology, vol. 43, no. 3, pp. 194-201, 2014.

[7] S. Sarkar and D. A. Fox, "Targeting il-17 and th17 cells in rheumatoid arthritis," Rheumatic Disease Clinics of North America, vol. 36, no. 2, pp. 345-366, 2010.

[8] M. Chabaud, J. M. Durand, N. Buchs et al., "Human interleukin17: a T cell-derived proinflammatory cytokine produced by the rheumatoid synovium," Arthritis and Rheumatism, vol. 42, no. 5, pp. 963-970, 1999.

[9] B. W. Kirkham, M. N. Lassere, J. P. Edmonds et al., "Synovial membrane cytokine expression is predictive of joint damage progression in rheumatoid arthritis: a two-year prospective study (the DAMAGE study cohort)," Arthritis and Rheumatism, vol. 54, no. 4, pp. 1122-1131, 2006.

[10] M. Ziolkowska, A. Koc, G. Luszczykiewicz et al., "High levels of IL-17 in rheumatoid arthritis patients: IL-15 triggers in vitro IL-17 production via cyclosporin A-sensitive mechanism," The Journal of Immunology, vol. 164, no. 5, pp. 2832-2838, 2000.

[11] W. Hueber, D. D. Patel, T. Dryja et al., "Effects of AIN457, a fully human antibody to interleukin-17A, on psoriasis, rheumatoid arthritis, and uveitis," Science Translational Medicine, vol. 2, no. 52, pp. 52r-72r, 2010.

[12] L. L. Lanier, C. Chang, and J. H. Phillips, "Human NKR-P1A: a disulfide-linked homodimer of the C-type lectin superfamily expressed by a subset of NK and T lymphocytes," The Journal of Immunology, vol. 153, no. 6, pp. 2417-2428, 1994.

[13] L. Cosmi, R. De Palma, V. Santarlasci et al., "Human interleukin 17-producing cells originate from a CD161+CD4+ T cell precursor," Journal of Experimental Medicine, vol. 205, no. 8, pp. $1903-$ 1916, 2008.

[14] L. Maggi, V. Santarlasci, M. Capone et al., "CD161 is a marker of all human IL-17-producing T-cell subsets and is induced by RORC," European Journal of Immunology, vol. 40, no. 8, pp. 2174-2181, 2010.

[15] P. Chalan, B. J. Kroesen, K. S. M. van der Geest et al., "Circulating CD4+CD161+ T lymphocytes are increased in seropositive arthralgia patients but decreased in patients with newly diagnosed rheumatoid arthritis," PLOS ONE, vol. 8, no. 11, Article ID e79370, 2013.

[16] J. Miao, J. Geng, K. Zhang et al., "Frequencies of circulating IL17-producing CD4+CD161+ T cells and CD4+CD161+ T cells correlate with disease activity in rheumatoid arthritis," Modern Rheumatology, vol. 24, no. 2, pp. 265-570, 2014.

[17] F. C. Arnett, S. M. Edworthy, D. A. Bloch et al., “The American Rheumatism Association 1987 revised criteria for the classification of rheumatoid arthritis," Arthritis and Rheumatism, vol. 31, no. 3, pp. 315-324, 1988.

[18] M. A. Kleinschek, K. Boniface, S. Sadekova et al., "Circulating and gut-resident human Th17 cells express CD161 and promote intestinal inflammation," Journal of Experimental Medicine, vol. 206, no. 3, pp. 525-534, 2009.

[19] A. Poggi, P. Costa, M. R. Zocchi, and L. Moretta, "Phenotypic and functional analysis of CD4+ NKRP1A+ human T lymphocytes. Direct evidence that the NKRP1A molecule is involved in transendothelial migration," European Journal of Immunology, vol. 27, no. 9, pp. 2345-2350, 1997.

[20] P. Chalan, B. J. Kroesen, K. S. M. van der Geest et al., "Circulating CD4+CD161+ $\mathrm{T}$ lymphocytes are increased in seropositive arthralgia patients but decreased in patients with 
newly diagnosed rheumatoid arthritis," PLoS ONE, vol. 8, no. 11, Article ID e79370, 2013.

[21] A. Poggi, P. Canevali, M. Contatore, and G. Ciprandi, "Higher frequencies of CD161+ circulating $\mathrm{T}$ lymphocytes in allergic rhinitis patients compared to healthy donors," International Archives of Allergy and Immunology, vol. 158, no. 2, pp. 151-156, 2012.

[22] A. Mitsuo, S. Morimoto, Y. Nakiri et al., "Decreased CD161+CD8+ $\mathrm{T}$ cells in the peripheral blood of patients suffering from rheumatic diseases," Rheumatology, vol. 45, no. 12, pp. 1477-1484, 2006.

[23] J. C. Crispín and G. C. Tsokos, "Human TCR- $\alpha \beta+$ CD4$\mathrm{CD} 8-\mathrm{T}$ cells can derive from CD8+ $\mathrm{T}$ cells and display an inflammatory effector phenotype," The Journal of Immunology, vol. 183, no. 7, pp. 4675-4681, 2009.

[24] J. C. Crispín, M. Oukka, G. Bayliss et al., "Expanded double negative $\mathrm{T}$ cells in patients with systemic lupus erythematosus produce IL-17 and infiltrate the kidneys," Journal of Immunology, vol. 181, no. 12, pp. 8761-8766, 2008.

[25] A. Alunno, O. Bistoni, E. Bartoloni et al., "IL-17-producing CD4-CD8- $\mathrm{T}$ cells are expanded in the peripheral blood, infiltrate salivary glands and are resistant to corticosteroids in patients with primary Sjögren's syndrome," Annals of the Rheumatic Diseases, vol. 72, no. 2, pp. 286-292, 2013. 


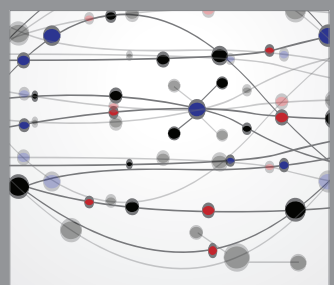

The Scientific World Journal
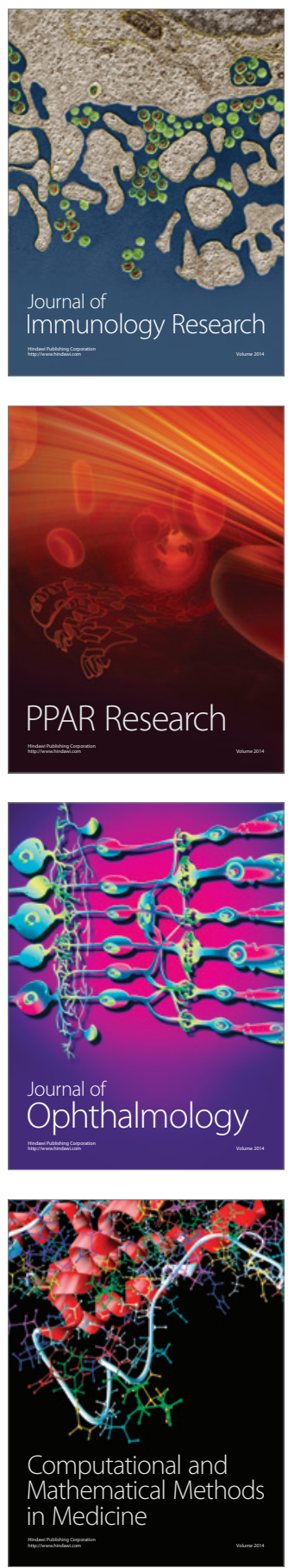

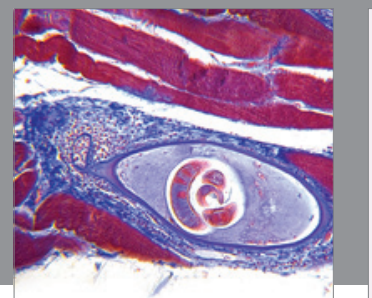

Gastroenterology

Research and Practice
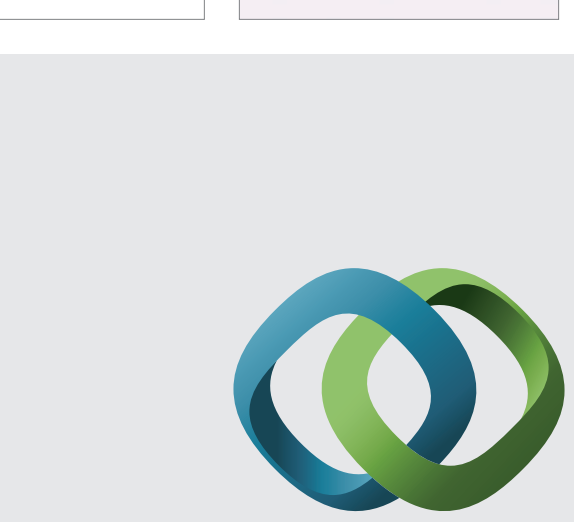

\section{Hindawi}

Submit your manuscripts at

http://www.hindawi.com
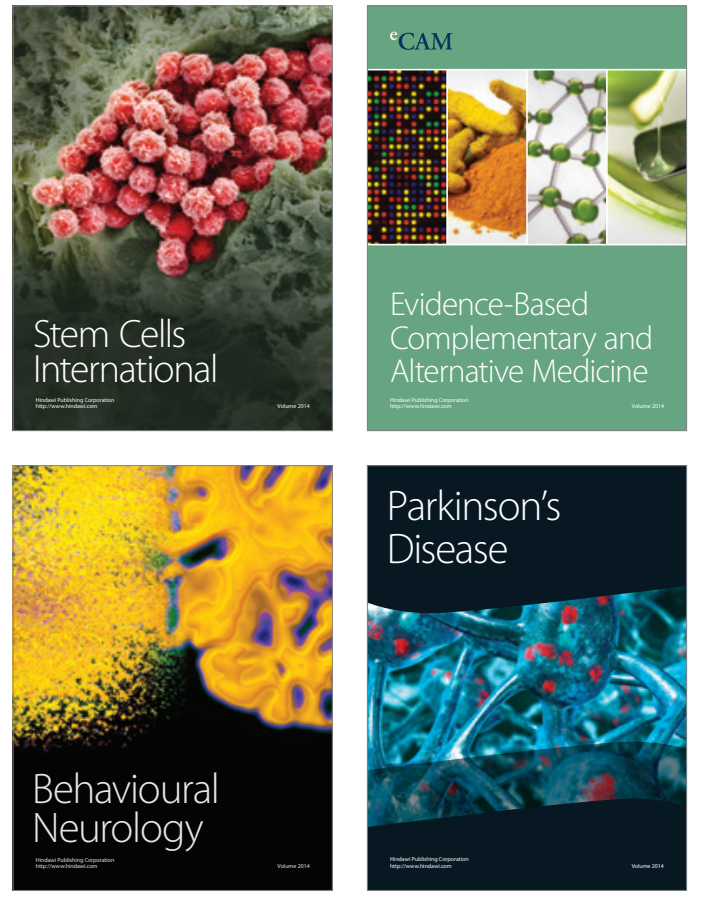
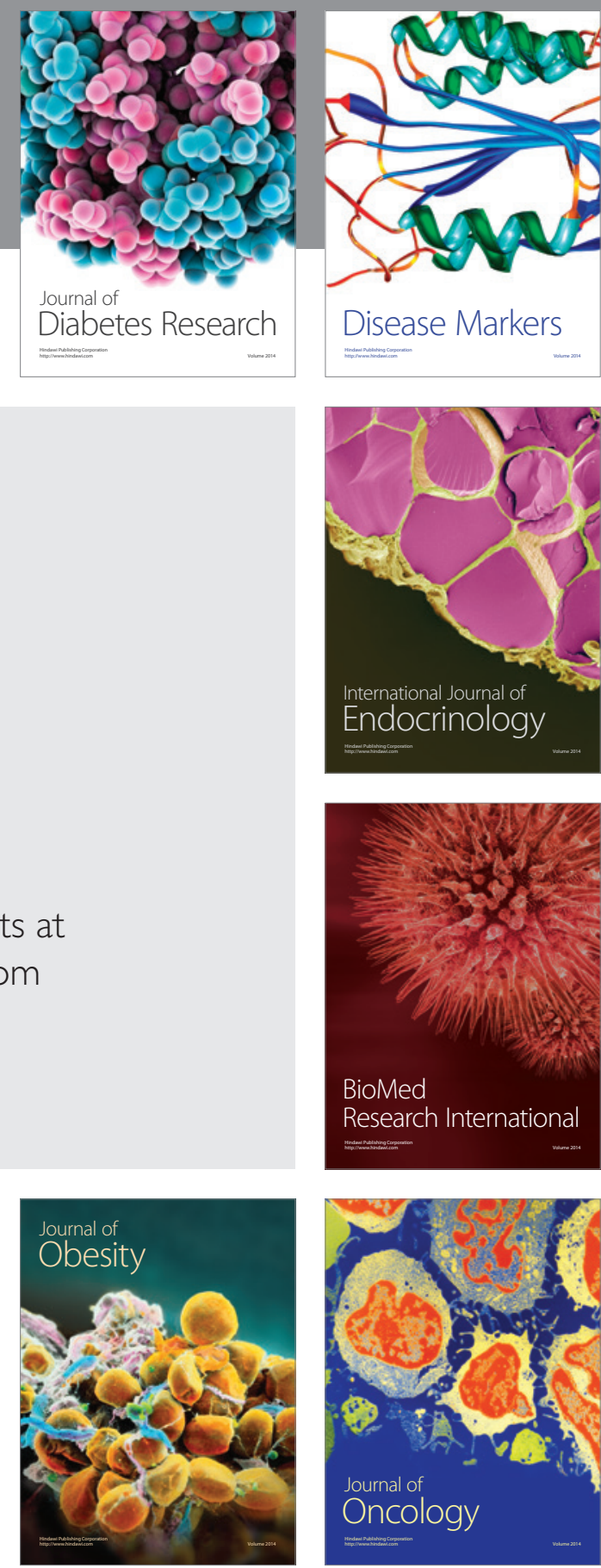

Disease Markers
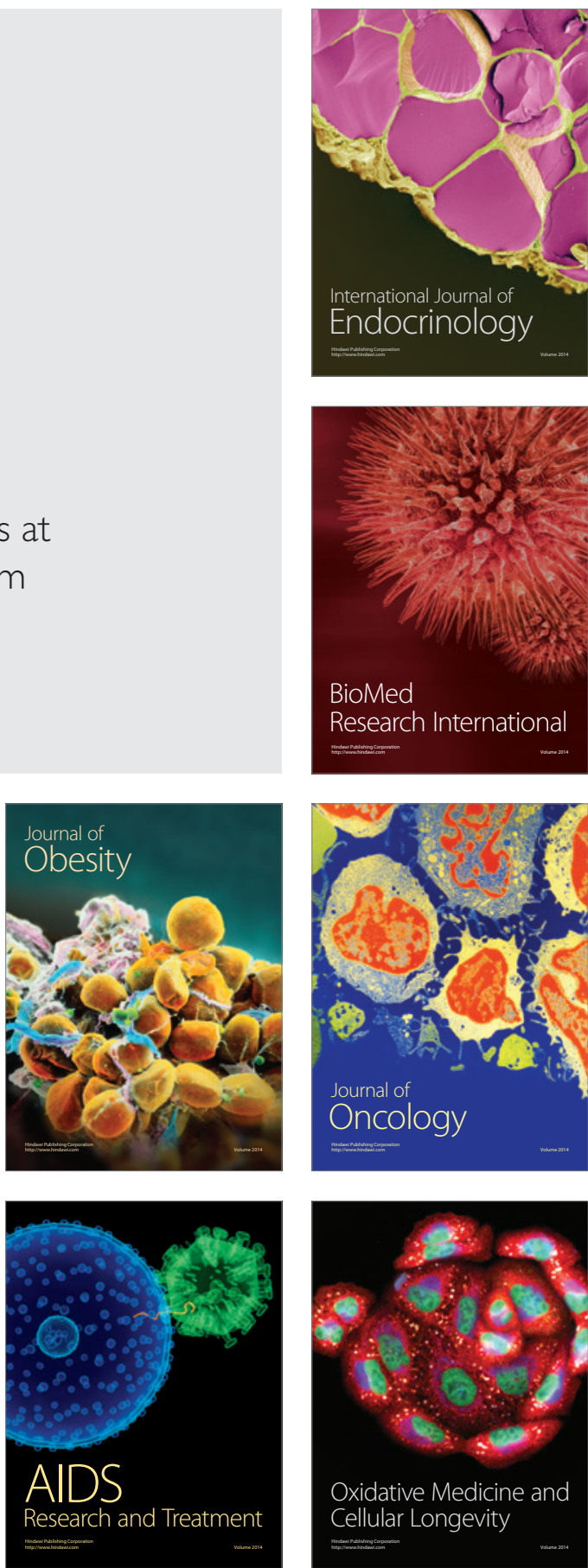